\title{
Bei uns kann Sterben unerträglich sein
}

\author{
Todeswünsche, auch Wünsche nach einem begleiteten Freitod, sollten besonders \\ im hohen Alter offen diskutiert werden. Selbstbestimmung darf auch am Lebens- \\ ende nicht vorenthalten werden. Zudem sollten Spitäler verpflichtet werden, einen \\ Teil ihrer Ärzte in Palliativmedizin zu schulen.
}

\section{Erika Preisig}

Dr. med., Hausärztin und Präsidentin des Vereins lifecircle; Autorin des Buches «Vater, du darfst sterben», Plädoyer einer Ärztin für den begleiteten Freitod; Coproduzentin der DVD «Notausgang», Perspektiven betreffend den begleiteten Freitod.

Weitere Informationen unter www.lifecircle.ch oder über mail[at]lifecircle.ch
Korrespondenz: Dr. med. Erika Preisig Hausarztpraxis bim Brunne Langgartenstrasse 2 CH-4105 Biel-Benken

e.preisig[at]sunrise.ch
Im Dezember dieses Jahres werden 30 Jahre vergangen sein, seit ich mein Staatsexamen in Basel bestanden habe. Ich liebe meinen Beruf und betreue seit fast 30 Jahren meine Patienten mit viel Hingebung. Ich habe Schönes und Schwieriges erlebt, wie dies in allen Berufen der Fall ist. Schon einmal habe ich in der S̈̈Z über einen meiner Patienten geschrieben, weil mich sein Schicksal unendlich beschäftigte und ich dieses Erlebnis mit meinen Berufskolleg(inn)en teilen wollte.

Heute schreibe ich einen zweiten Verlaufsbericht über drei Schicksale, die mich als Ärztin sehr betroffen machen. Ich möchte anhand dieser Schicksale meine Berufskolleg(inn)en und ganz besonders die Spitalärzt(inn)en sowie Behörden aufrütteln.

Vor einigen Monaten rief mich der Neffe einer 96-jährigen Frau an. Sie war im Alter von 94 Jahren Mitglied von Exit geworden mit der klaren Absicht, ihr bisher völlig autonomes Leben im Falle einer schweren Erkrankung mit Exit zu beenden. Im Frühjahr 2014 erlitt sie einen Herzinfarkt. Nach einer langen Zeit der Reha stand fest, dass die Frau schwerst pflegebedürftig bleiben würde und ins Pflegeheim verlegt würde. Darauf eröffnete die Patientin dem Chefarzt, sie brauche nicht ein Pflegeheim, sondern Exit. Der Chefarzt aber rief den Gerontopsychiater, anstatt Exit. Dieser diagnostizierte eine Depression, und somit eingeschränkte Urteilsfähigkeit. Die Folge war, dass die Patientin ihren Wunsch nach einem begleiteten Freitod nicht realisieren konnte und im Pflegeheim noch lange hätte weiterleben müssen, in einem Zustand, den sie mit der Mitgliedschaft bei Exit verhindern wollte.

Hier stellen sich folgende Fragen:

- Ist es richtig, einen Menschen wegen seines Todeswunsches als depressiv zu erklären?

- Verliert ein Mensch seine Urteilsfähigkeit, wenn er depressiv ist?

Der Neffe gab nicht auf, wandte sich an mich, da er vernommen hatte, dass ich in Basel eine neue Organisation gegründet hatte, die sich für den begleiteten Freitod einsetzt. Ich ging noch am gleichen Tag ins Spital zu der Patientin. Nach einem sehr langen Gespräch war mir klar, dass diese Frau zu 100\% ur-

\section{Dans notre canton, mourir peut encore être un calvaire}

Cet article se penche sur trois cas de demandes de mort assistée (MA):

- Une femme de 96 ans, jugée dépressive après avoir demandé la MA. La dépression prive-t-elle un individu de sa capacité de discernement? A-t-on réellement besoin d'un psychiatre lorsque l'on demande la MA à cet âge?

- Une femme polymorbide de $\mathbf{8 6}$ ans demande la MA. Je propose la sédation terminale (ST) au lieu de la MA, parce que le transport au domicile aurait été inhumain. Mais la ST n'est pas appliquée par l'hôpital. La femme meurt en criant de douleur.

- Un homme souffrant de démence demande la MA. Grâce à la compréhension des médecins d'une clinique spécialisée dans la démence, ce patient a pu bénéficier d'une MA en présence de ses proches.

Forte de mon expérience, je reproche aux médecins et aux hôpitaux suisses de n'être pas suffisamment formés aux soins palliatifs et de ne pas accepter la MA alors que l'association EXIT compte plus de $\mathbf{8 5 0 0 0}$ membres.

En tant que médecin généraliste, je demande que l'on introduise sans attendre dans chaque hôpital non seulement les soins palliatifs mais également la MA. A cet égard, les propos du Prof. Scheidegger, ancien chef des soins intensifs de l'Hôpital universitaire de Bâle, actuellement retraité, méritent réflexion: «Si nous laissions mourir les gens qui veulent mourir, nous aurions assez d'argent pour ceux qui veulent vivre.»

teilsfähig und keinesfalls depressiv war. Ich konnte den Entscheid des Psychiaters nicht nachvollziehen. Dem Neffen empfahl ich, mit dem Chefarzt und dem Psychiater das Gespräch zu suchen und bot an, dabei behilflich zu sein. 


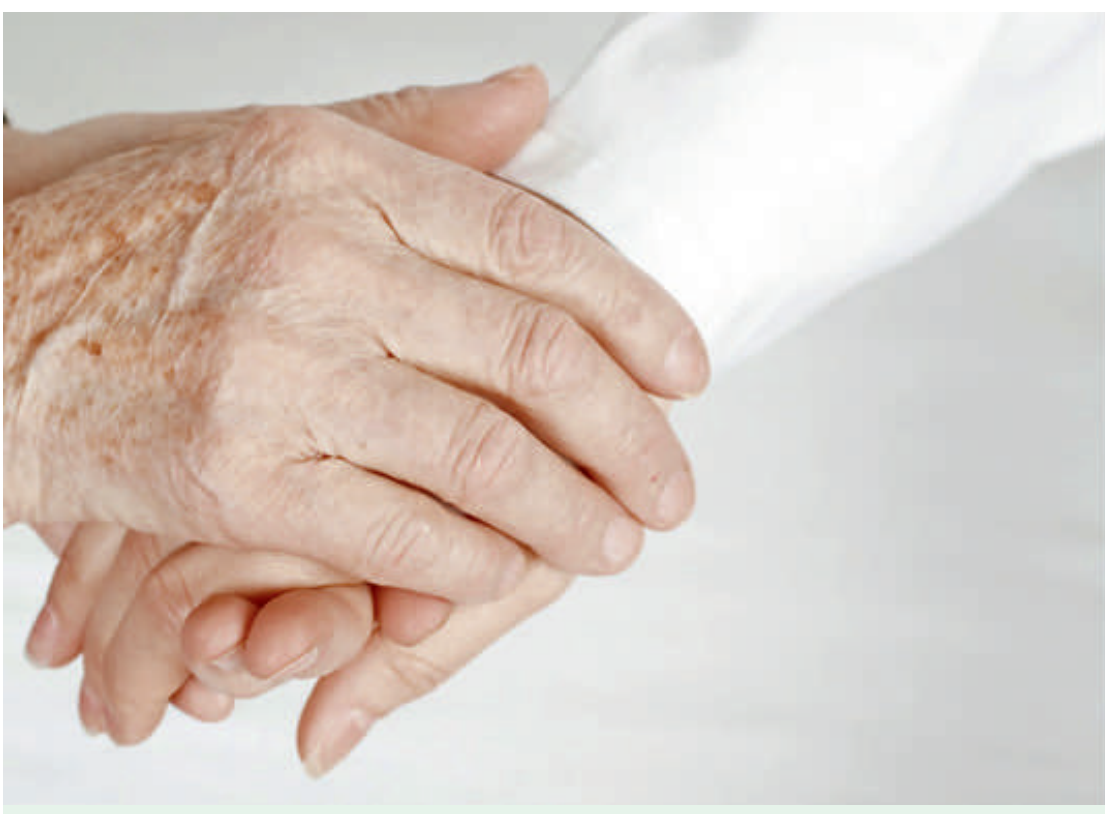

Patienten bekommen von Ärzten nicht immer die Hilfe, die sie sich erhoffen.

Kurz darauf fand das Gespräch statt. Es war ein Ringen um das Selbstbestimmungsrecht der Patientin. Erst als ich den Psychiater auf wirtschaftliche Interessen am Weiterleben der Patientin ansprach, war er zu einer erneuten Beurteilung der Patientin bereit. Zwei Tage später schlief die Frau glücklich und in Anwesenheit ihres Neffen und dessen Lebenspartners für immer ein, durch eigenhändiges Öffnen einer Infusion mit Natrium Pentobarbital in tödlicher Dosis. Nicht im Spital, sondern in der Wohnung der Stiftung Eternal Spirit in Basel.

Mitte Juni erhielt ich wiederum einen Hilferuf von einem Mann, dem Sohn einer 86-jährigen Frau. Sie lag mit einer Gangrän im Spital, man hätte den Unterschenkel amputieren müssen, um die Patientin zu retten. Die Frau entschied sich aber gegen eine Operation, und bat um einen begleiteten Freitod.
Spirit möglich wäre. Am folgenden Tag fand ein Familiengespräch mit der Assistenzärztin statt, währenddessen ich mir ein Bild der Situation machen konnte. Die Patientin war absolut klar in ihrem Todeswunsch, aber in einem so desolaten Zustand, dass ihr insbesondere durch die schmerzhafte Gangrän ein Heimtransport nicht zugemutet werden konnte.

Ich schlug vor, anstatt des begleiteten Freitodes in diesem Falle eine terminale Sedation (TS) einzuleiten.

Wäre es möglich gewesen, ihr im Spital ohne Transport einen begleiteten Freitod zu ermöglichen, hätte ich dies gerne baldmöglichst getan. In der Annahme, dass es in jedem Spital möglich ist, eine TS durchzuführen, versprach ich der Frau, dass sie zwar nicht den gewünschten begleiteten Freitod, wohl aber ein schmerzloses Hinübergleiten durch eine Sedation erhalten könne.

Warum eine TS bei der Patientin nicht durchgeführt werden konnte, ob es an Wissen um Palliativmedizin oder eher an mangelnder Kommunikation zwischen den Stationen fehlte, war im Gespräch mit den zuständigen Chefärzten nicht klar.

Die Patientin hat laut Aussagen der Angehörigen weitere 5 Tage unendlich gelitten. Die Schmerzmittel wurden oft erst gespritzt, nachdem Schmerzspitzen schon längst erreicht waren. Immer wieder haben die anwesenden Verwandten um Schmerzmittel gebeten. Bis dann die Pflegefachfrau endlich einen Arzt gefunden und von ihm eine höhere Dosis bewilligt erhielt, sei laut Aussagen der anwesenden Verwandten jeweils eine unhaltbare Situation aufgetreten.

Unnötiges Leiden und allenfalls finanzielle Interessen des Spitals an der im Erstklasszimmer liegenden Patientin, das sind die Anschuldigungen, die mir die ununterbrochen anwesenden Verwandten nach dem Ableben der Patientin geschildert haben. Wenn ich auch nur die Hälfte der Details glaube, die ich da gelesen habe, entstehen bei mir grosse Ängste, dass ich selber einmal in einem Spital derartig ausgeliefert mein Leben beenden muss.

\section{«Warum gibt es keine Vorschriften, dass jedes Spital eine gewisse Anzahl an Ärzten in Palliativmedizin fortbilden muss?»}

Die behandelnden Ärzte konnten den Todeswunsch der Patientin nachvollziehen, stellten dem Sohn ein Rezept für das notwendige Medikament in Aussicht. Der Austritt aus dem Spital und die Übergabe des Rezeptes an den Sohn war auf den Mittwoch geplant. Beides fand aber nicht statt. Die Ausstellung des Rezeptes durch die Spitalärzte war plötzlich nicht mehr möglich, Gründe dafür wurden den Angehörigen nicht angegeben.

In seiner Verzweiflung bat mich der Sohn, seine Mutter im Spital zu besuchen und zu entscheiden, ob ein begleiteter Freitod durch die Stiftung Eternal
Auch hier stellen sich Fragen:

- Warum kann ein Spital Palliativmedizin nicht so anwenden, dass die terminal kranke Frau sediert und absolut ohne Schmerzen hätte sterben können?

- Warum gibt es keine Vorschriften, dass jedes Spital eine gewisse Anzahl an Ärzten in Palliativmedizin fortbilden muss, damit solche Situationen nicht vorkommen?

- Warum muss eine unheilbar schwerkranke Person das Spital verlassen, um einen begleiteten Freitod realisieren zu können? 
- Wie viele Menschen müssen noch aus Fenstern und von Balkonen der Spitäler springen, bevor die Spitäler den begleiteten Freitod akzeptieren?

Der dritte Fall betrifft einen Patienten eines Berufskollegen, der zwar selber keinen begleiteten Freitod ermöglicht, aber den Todeswunsch seiner Patienten respektiert. Er spricht mit ihnen offen darüber, und leitet sie an mich weiter, falls er den Todeswunsch nachvollziehen kann. Der betreffende Patient war an einer Alzheimer-Demenz erkrankt. Im September 2013 war er in der Memory-Klinik beurteilt worden, aber die Krankheit schritt ab Januar 2014 schnell voran. Als ich den Mann in seiner Wohnung besuchte, war er noch klar urteilsfähig, aber es war deutlich erkennbar, dass er im fortgeschrittenen Stadium an seinem Morbus Alzheimer litt. Wollte man einen begleiteten Freitod verantworten, musste dieser bald stattfinden. Aber die Urteilsfähigkeit musste sicher noch einmal von einem Neurologen, idealerweise von der Memory-Klinik beurteilt werden. Ich rief den Chefarzt der Memory-Klinik an und erklärte ihm mein Anliegen. Er teilte mir mit, dass sie eine Warteliste von bis zu drei Monaten hätten. Für den Betroffenen hätte dies bedeutet, dass er «den Zug verpasst» hätte. Drei Monate später wäre er höchst wahrscheinlich in einer geschlossenen Institution untergebracht gewesen, so wie er dies für sich nie wollte.

Auf meinen Hinweis, dass mit 70000 Exit-Mitgliedern und weiter steigender Lebenserwartung immer mehr Menschen ähnliche Bedürfnisse äussern werden wie dieser Herr, sicherte mir der Chefarzt zu, er werde die Situation mit dem hauptverantwortlichen Psychiater diskutieren. Eine Woche später hatte der Herr einen Termin in der Memory-Klinik und wurde dort in Bezug auf seinen Todeswunsch als urteilsfähig erklärt. Wenige Tage später schlief er mittels Natrium Pentobarbital in Anwesenheit seiner Tochter und seines Bruders sehr dankbar und friedlich ein. Wir alle waren uns bewusst, dass ich ohne den kurzfristig ermöglichten Termin in der MemoryKlinik das Risiko, ihm den begleiteten Freitod zu ermöglichen, nicht hätte eingehen können.

$\mathrm{Zu}$ diesem Fall möchte ich der Memory-Klinik meinen grössten Dank aussprechen, dass diese Ärzte den Mut und die Grosszügigkeit hatten, den Todeswunsch des Mannes zu respektieren und seine Urteilsfähigkeit zu attestieren. So konnte er sich seinen sehnlichsten Wunsch erfüllen, nicht noch mehrere Jahre in einer geschlossenen Institution weiter sein Dasein fristen zu müssen. kommentieren? Nutzen Sie dafür die Kommentarfunktion in der OnlineVersion oder sehen Sie nach, was Ihre Kolleginnen und Kollegen bereits geschrieben haben: www.saez.ch/ aktuelle-ausgabe/ interaktive-beitraege/

\section{im Kanton Basel-Landschaft}

Im Kanton Basel-Landschaft haben wir ein ausgezeichnetes Hospiz mit 12 Plätzen. Unser kleinstes Kantonsspital arbeitet mit der spitalexternen Onkologiepflege zusammen, einem Team, das jederzeit zur Verfügung steht und sehr gute Palliativmedizin ausübt, auch allen Hausärzten zur Verfügung. Eines der grösseren Kantonsspitäler hat seit Januar 2014 ein spitalinternes Palliativteam, es sind zwei Einzelzimmer für Palliativfälle reserviert. Ein weiteres Kantonsspital hat eine Onkologin, die sich in Palliativmedizin weiterbildet und für Fälle wie bei der alten Dame zur Verfügung steht. Zudem haben sich zwei anthroposophische Kliniken zusammengeschlossen. Diese sind bereit, Palliativmedizin anzubieten, haben aber wie alle andern Klinken Probleme, da es noch keine DRG-Position gibt, welche die effektiven Kosten einer palliativmedizinischen Betreuung dann auch deckt.

Man könnte meinen, ein «Fall» wie die Patientin mit der Gangrän müsste nicht vorkommen. Tatsache ist, dass sich auch in Spitälern mit mehreren Hundert Betten oft nur ein einziger Arzt in Palliativmedizin weitergebildet hat und somit hoffnungslos überfordert ist. Zudem hat dieser Arzt allenfalls noch nicht genügend palliativmedizinische Erfahrung, um auch eine terminale Sedation zu verantworten. Im Kanton Basel-Landschaft sterben pro Jahr 2300 Menschen, $85 \%$ davon in Spitälern und Altersheimen ohne gute palliativmedizinische Betreuung und ohne die Möglichkeit, ihr Recht auf einen begleiteten Freitod ausüben zu können.

Ich schäme mich als Schweizer Ärztin, dass wir es nicht fertigbringen, mehr Palliativplätze anzubieten.

Herr Professor Scheidegger, pensionierter Chefarzt der Intensivstation des Universitätsspitals Basel, hat kürzlich mit mir auf einem Podium der CVP diskutiert. Er hatte den Mut, Folgendes zu sagen: «Wenn wir die Menschen, die ihr Leiden nicht mehr tragen wollen, und konstant ihren Todeswunsch ausdrücken, gehen lassen würden, und wenn wir von allen diesen Wunsch kennen würden, bevor sie nach einem Eingriff auf der Intensivstation landen, könnten wir viel unnötiges Leiden verhindern, könnten viel Geld sparen und hätten dieses Geld für diejenigen zur Verfügung, die leben wollen.»

Meine sehnlichsten Wünsche als Hausärztin und Präsidentin der Lebenshilfeorganisation lifecircle und der Sterbehilfeorganisation Stiftung Eternal Spirit sind:

- Spitäler sollten verpflichtet werden, einen Teil ihrer Ärzte in Palliativmedizin zu schulen, auch Hausärzte müssten sich in Palliativmedizin weiterbilden. - Die zuständigen Gremien müssen die DRG für Palliativmedizin innerhalb von Wochen, nicht Monaten regeln, Palliativmedizin ist für Spitäler sonst nicht wirtschaftlich und wird somit kaum ausgeübt.

- Todeswünsche, auch Wünsche nach einem begleiteten Freitod, sollten ganz besonders im hohen Alter offen diskutiert werden, den Menschen darf die Selbstbestimmung auch am Lebensende nicht vorenthalten werden. inklusive der Möglichkeit einer TS. Dieses Team steht
- Chefärzte sollten zusammensitzen und eine Lösung finden, damit zumindest bei terminal Kranken mit Wunsch nach einem begleiteten Freitod rechtzeitig Exit oder lifecircle genauso wie Palliativmediziner zugezogen werden. 